\title{
On the Influence of Bottom Topography on Ocean Currents.
}

\author{
By \\ Kinjiro KAJIURA \\ (Geophysical Institute, Tokyo University)
}

\begin{abstract}
Topographic effect on currents has been discussed by the method of small perturbation. An equation for the vertical motion is derived which includes a wide range of phenomena, and it is shown that the character of the deflection of currents by the bottom deformation is complicated since the different kinds of waves become dominant according to the scale of topography, and, moreover, since the horizontal shear of a current takes part in the phenomena. When the scale of the bottom deformation is very small, the stationary internal gravity waves prevail under a certain condition and when the scale is large, planetary waves develop in a horizontal plane.
\end{abstract}

\section{Introduction}

It is a well known fact that the distribution of density is considerably modified when an ocean current flows across a submarine ridge. Theoretical attacks to this problem, however, are very scarce owing to difficulties of mathematical analyses. In his very extensive studies on the character of ocean currents in homogeneous water, V. W. Ekman (1923) first examined the effect of bottom topography on slope currents, the velocity of which is constant with depth except in a layer influenced by the bottom friction. His conclusions are: The currents generally tend to follow the bottom contours in higher latitudes and in the northern hemisphere, they deviate to the right, passing over an elevation and to the left, over depressions of the bottom, and vice versa in the southern hemisphere. He extended his analysis later (1932) on the basis of hydrodynamic equations including inertial terms as well as frictional terms, but the major features were almost as before. Similar studies of the problem have been made by many meteorologists concerning to an air flow over a mountain. P. Queney $(1947,1948)$ has developed an extensive study of perturbations of air flow and discussed the mountain effect, taking the vertical stability of the atmosphere into account. Recently, the influence of a large scale mountain range on westerlies has been discussed by many authors ( $\mathrm{J}$. Charney and A. Eliassen, 1949, D. Colson, 1949, B. Bolin, 1950, etc.) on the basis of the vorticity equation. In these studies, frictional effects are neglected and the general flow 
is assumed to be zonal and uniform. According to the vorticity equation in a barotropic atmosphere, the horizontal divergence or convergence due to the effect of topography results in an increase or decrease of vorticity, $r$ espectively, and the deflection of stream lines can be expected on the downstream side which is followed by the planetary waves of Rossby type.

On the other hand, the effect of bottom topography on relative current in non-homogeneous water has been examined qualitatively by $H$. U. Sverdrup (1941). The conclusion of his analysis is that the observed deflections of currents, which seem to be maximum at the summit of the bottom ridge, may not be caused by bottom friction as was assumed by Ekman, but they are caused by modifications of the density distribution due to the effect of topography. In his theory, the assumption that the isopicnic surface must rise when a watermass approaches a ridge, includes essentially the existence of a vertical velocity as well as a horizontal velocity near the bottom, and this is inconsistent with the "law of the parallel solenoids" which is the fundamental concept in his discussion.

In these circumstances, V. B. Stockman (1947, 1948) reviewed the former studies and discussed the topographic effect, using the concepts of mass transport and lateral frictions. He emphasized the primary importance of the distribution of wind system over the ocean which is the main cause of ocean currents, and concluded that the deflection of currents by the bottom topography may not be related to the Coriolis force, but is connected with the vorticity in the windfield, such that when a current crosses a sea-bottom elevation, the flow-lines deviate over its summit in the same direction as the vorticity in the wind field and just the opposite above the central part of a depression. Though his conclusions are very suggessive, his deduction of results seems to be very doubtful in respect to the procedure of simplifying the equations of motion, by means of taking mean values of various quantities.

At any rate, it is a usual experience that the currents, flowing close to the coast or flowing slowly, are apt to follow the bottom contours. It is, however, not so exactly ascertained that in which direction they will turn when ocean currents pass over an elevation or depression of the bottom. Therefore, in the present paper, the effect of bottom topography on ocean currents has been reexamined on the basis of the hydrodynamic equations including inertial terms, taking the density distribution into consideration as far as possible. 


\section{Basic Equations of Motion.}

We assume that the field is stationary and may be divided into two parts, of which one is the basic field and the other is the field of small parturbations caused by irregularities of the bottom. In spite of the defects involved in the approximation of small perturbations, the qualitative features of a flow pattern may be estimated. We write the pressure, $p=p_{0}+p_{1}$, the density $\rho=\rho_{0}+\rho_{1}$, the horizontal components of velocity $u=u_{0}+u_{1}, v=v_{1}$ and the vertical component of velocity $u=w_{1}$, where subscripts 0 and 1 refer to the quantities in the basic state and the disturbed state, respectively.

The basic current is assumed geostrophic and zonal defined by the following equations :

$$
\rho_{0} \frac{\partial p_{0}}{\partial y}=-\lambda u_{0}
$$

and

$$
\frac{\partial p_{0}}{\rho_{0}}=-g
$$

where the co-ordinate $x, y$ and $z$ are directed to the east, to the north and vertically upward, respectively, with the origin situated at the bottom, and $\lambda(=2 \omega \sin \varphi)$ is the Coriolis parameter and $g$ is the acceleration due to gravity.

The equations of motion for stationary perturbations are:

$$
\begin{aligned}
u_{0} \frac{\partial u_{1}}{\partial x}+v_{1} \frac{\partial u_{0}}{\partial y}+w_{1} \frac{\partial u_{0}}{\partial z} & =-\frac{1}{\rho_{0}} \frac{\partial p_{1}}{\partial x}+\lambda v_{1}, \\
u_{0} \frac{\partial v_{1}}{\partial x} & =-\frac{1}{\rho_{0}} \frac{\partial p_{1}}{\partial y}-\frac{\rho_{1}}{\rho_{0}} \lambda u_{0}-\lambda u_{1},
\end{aligned}
$$

and

$$
u_{0} \frac{\partial w_{1}}{\partial x} \quad=-\rho_{\rho_{0}}^{1} \frac{\partial p_{1}}{\partial z}-\frac{\rho_{1}}{\rho_{0}} g
$$

provided that the frictional and other small terms be neglected and that the basic current $u_{0}$ is assumed to be independent of $x$.

When the fluid is incompressible and individual density is conservative, we have:

$$
\frac{\partial u_{1}}{\partial x}+\frac{\partial v_{1}}{\partial y}+\frac{\partial w_{1}}{\partial z}=0
$$

and

$$
u_{0} \frac{\partial \rho^{\prime}}{\partial x}+v_{1} \frac{\partial \rho_{0}}{\partial y}+w_{1} \frac{\partial \rho_{0}}{\partial z}=0
$$


Cross differentiating (3) and (4) with respect to $x$ and $y$, and taking (6) and $(7)$ into account, we obtain:

$$
\begin{aligned}
& \frac{\partial^{2} v_{1}}{\partial x^{2}}+\frac{\partial^{2} v_{1}}{\partial y^{2}}+\left(\frac{1}{\rho_{0}} \frac{\partial \rho_{0}}{\partial y}\right) \frac{\partial v_{1}}{\partial y}+\left\{\frac{1}{u_{0}} \frac{\partial}{\partial y}\left(\lambda-\frac{\partial u_{0}}{\partial y}\right)-\frac{1}{u_{0}} \frac{\partial u_{0}}{\partial y} \cdot \frac{1}{\rho_{0}} \frac{\partial \rho_{0}}{\partial y}\right\} v_{1} \\
& =-\frac{\partial^{2} w_{1}}{\partial y \partial z}+\left(\frac{1}{u_{0}} \frac{\partial u_{0}}{\partial z}\right) \frac{\partial w_{1}}{\partial y}+\left\{\frac{1}{u_{0}}\left(\lambda-\frac{\partial u_{0}}{\partial y}\right)-\frac{1}{\rho_{0}} \frac{\partial \rho_{0}}{\partial y}\right\} \frac{\partial w_{1}}{\partial z} \\
& +\left(\frac{1}{u_{0}} \cdot \frac{\partial^{2} u_{0}}{\partial y \partial z}+\frac{1}{u_{0}} \frac{\partial u_{0}}{\partial z} \cdot \frac{1}{\rho_{0}} \frac{\partial \rho_{0}}{\partial y}+\frac{\lambda}{u_{0}} \cdot \frac{1}{\rho_{0}} \frac{\partial \rho_{0}}{\partial z}\right) w_{1},
\end{aligned}
$$

and from (3) and (5), we have:

$$
\begin{aligned}
\frac{\partial^{2} w_{1}}{\partial x^{2}}+\frac{\partial^{2} w_{1}}{\partial z^{2}}+\left(\frac{1}{\rho_{0}} \frac{\partial \rho_{0}}{\partial z}\right) \frac{\partial w_{1}}{\partial z}-\left\{\left(\frac{1}{u_{0}} \frac{\partial u_{0}}{\partial z}+\frac{g}{u_{0}^{2}}\right) \frac{1}{\rho_{0}} \frac{\partial \rho_{0}}{\partial z}+\frac{1}{u_{0}} \frac{\partial^{2} u_{0}}{\partial z^{2}}\right\} w_{1} \\
=-\frac{\partial^{2} v_{1}}{\partial y \partial z}-\left(\frac{1}{u_{0}} \frac{\partial u_{0}}{\partial z}+\frac{1}{\rho_{0}} \frac{\partial \rho_{0}}{\partial z}\right) \frac{\partial v_{1}}{\partial y}-\frac{1}{u_{0}}\left(\lambda-\frac{\partial u_{0}}{\partial y}\right) \frac{\partial v_{1}}{\partial z} \\
-\left\{\frac{1}{u_{0}}\left(\lambda-\frac{\partial u_{0}}{\partial y}\right) \frac{1}{\rho_{0}} \frac{\partial \rho_{0}}{\partial z}-\frac{1}{u_{0}} \cdot \frac{\partial^{2} u_{0}}{\partial y \partial z}-\frac{g}{u_{0}} \cdot \frac{1}{\rho_{0}} \frac{\partial \rho_{0}}{\partial y}\right\} v_{1} .
\end{aligned}
$$

Eqs. (8) and (9) may be simplified when adequate assumptions are introduced.

When the horizontal gradient of density is very small and the basic current may be considered to be almost uniform*, we have, from ( 8 ), neglecting the variation of velocities in the $y$-direction,

$$
\frac{\partial^{2} v_{1}}{\partial x^{2}}+\frac{1}{U} \frac{\partial \lambda}{\partial y} v_{1}=\frac{\lambda}{U}\left(\frac{\partial w_{1}}{\partial z}+\frac{w_{1}}{\rho_{0}} \frac{\partial \rho_{0}}{\partial z}\right)
$$

and from $(9)$,

$$
\frac{\partial^{2} w_{1}}{\partial x^{2}}+\frac{\partial^{2} w_{1}}{\partial z^{2}}+\left(\frac{1}{\rho_{0}} \frac{\partial \rho_{0}}{\partial z}\right) \frac{\partial w_{1}}{\partial z}-\frac{g}{U^{2}}\left(\frac{1}{\rho_{0}} \frac{\partial \rho_{0}}{\partial z}\right) w_{1}=-\frac{\lambda}{U}\left(\frac{\partial v_{1}}{\partial z}+\frac{1}{\rho_{0}} \frac{\partial \rho_{0}}{\partial z} \cdot v_{1}\right),
$$

where $U$ is the uniform basic velocity.

Here we shall transform $v_{1}$ and $w_{1}$ into $v^{\prime}$ and $w^{\prime}$ by the following equations :

$$
v_{1}=\int_{-\infty}^{\infty} v^{\prime} e^{i k x} d k \text {, and } w_{1}=\int_{-\infty}^{\infty} w^{\prime} e^{i k x} d k
$$

* From equations (1) and (2), we have,

$$
u_{0}=U+\frac{g}{\lambda \rho_{0}} \int_{0}^{z} \frac{\partial \rho_{0}}{\partial y} d z \text {, and } U=-\frac{1}{\lambda \rho_{0}} \frac{\partial P_{0 B}}{\partial y},
$$

where $P_{0 B}$ is the bottom pressure. The assumption of almost uniform basic current is satisfied when the condition, $\left|\frac{\partial P_{0 B}}{\partial y}\right| \gg\left|g \int_{0}^{z} \frac{\partial \rho_{0}}{\partial y} d z\right|$, is fulfilled, in other words, when the baroclinic effect is neglected. Since the relative currents prevail in the ocean, this assumption can not be accepted. Our discussion, however, will be valid if the effect of the vertical shear of velocity does not play a dominant role. Thus, $U$ may be given, regardless of (1) and (2), in our discussion. 
Writting $\frac{1}{\rho_{0}} \frac{\partial \rho_{0}}{\partial z}=-s$ and $\frac{\partial \lambda}{\partial y}=\beta$, both of which are assumed to be constant, we have from (10) and (11), under the condition $\left|\frac{1}{w_{1}} \frac{\partial w_{1}}{\partial z}\right| \gg\left|\frac{1}{\rho_{0}} \frac{\partial \rho_{0}}{\partial z}\right|$ :

$$
v^{\prime}=\frac{k_{\lambda}}{k_{\beta}^{2}-k^{2}} \cdot \frac{\partial w^{\prime}}{\partial z},
$$

and

$$
\frac{\partial^{2} w^{\prime}}{\partial z^{2}}+\frac{\left(k_{s}^{2}-k^{2}\right)\left(k_{\beta}^{2}-k^{2}\right)}{\left(k_{\lambda}^{2}-k^{2}\right)} \cdot w^{\prime}=0,
$$

where $k_{\beta}{ }^{2}=\frac{\beta}{U}, k_{\lambda}{ }^{2}=\frac{\lambda^{2}}{U^{2}}, k_{s}{ }^{2}=\frac{g_{s}}{U^{2}}$, and the term containing $\frac{\partial w^{\prime}}{\partial z}$ is neglected, since $s$, the coefficient of $\frac{\partial w^{\prime}}{\partial z}$, is sufficiently small. The orders of magnitude of various parameters are tabulated in Table 1 . Here, we assume $k_{\beta}$ and $k_{s}$ to be positive, since we are chiefly concerned with steady and stable perturbations.

Table 1. Order of magnitude of various parameters.

\begin{tabular}{|c|c|c|c|c|}
\hline$U(\mathrm{~cm} / \mathrm{sec})$ & $k_{s}{ }^{2}\left(\mathrm{~cm}^{-2}\right)$ & $k_{\lambda}{ }^{2}\left(\mathrm{~cm}^{-2}\right)$ & $k_{\beta}{ }^{2}\left(\mathrm{~cm}^{-2}\right)$ \\
& $s=10^{-8}$ & $s=10^{-6}$ & $\lambda=10^{-4}$ & $\beta=10^{-13}$ \\
1 & $10^{-5}$ & $10^{-3}$ & $10^{-5}$ & $10^{-13}$ \\
10 & $10^{-7}$ & $10^{-5}$ & $10^{-10}$ & $10^{-14}$ \\
100 & $10^{-9}$ & $10^{-7}$ & $10^{-12}$ & $10^{-15}$ \\
\hline
\end{tabular}

In (14), we can see that when $k \gg k_{\beta}, k_{\beta}^{2}$ may be neglected and the equation will hold for any direction of a basic current.

\section{Solution of the equations.}

A formal solution of (14) is,

$$
w^{\prime}=A e^{i p z}+B e^{-i p z}
$$

where $p^{2}=\left(k_{s}{ }^{2}-k^{2}\right)\left(k_{\beta}^{2}-k^{2}\right) /\left(k_{\lambda}{ }^{2}-k^{2}\right)$ and $A$ and $B$ are constants of integration. Since $p^{2}$ is positive when $k_{s}>k>k_{\lambda}$ or $k<k_{\beta}$ and negative when $k>k_{s}$ or $k_{\lambda}>k$ $>k_{\beta}, p$ is real or pure imaginary according to the value of $k$. Substituting (15) into (12), we have,

$$
w_{1}=\int_{-\infty}^{\infty}\left(A e^{i p z}+B e^{-i p \bar{z}}\right) e^{i k x} d k
$$

Here, boundary conditions for the vertical velocity $u_{1}$ are given by

$$
w_{1}=U \frac{\partial h}{\partial x}, \text { at the bottom, }
$$

and 


$$
w_{1}=0, \quad \text { at the surface, } ;
$$

where $h$ is the height of the bottom deformation which is assumed to be independent of $y$ corresponding to the assumption that the velocity variation in $y$-direction is negligible.

In the case when the bottom deformation is expressed by $h=h_{0} \cos k x$ and the mean depth is $H$, we have from (16), (17) and (18), as a particular solution:

$$
w_{1}=-\frac{U k h_{0}}{\sin p H} \sin p(H-z) \sin k x ;
$$

from (10) and (19),

$$
v_{1}=\frac{U k h_{0}}{\sin p H} \cdot \frac{k_{\lambda}}{k_{\beta}^{2}-k^{2}} \cdot p \cos p(H-z) \sin k x
$$

from $(6),(19)$ and $(20)$,

$$
u_{1}=\frac{U h_{0} p}{\sin p H} \cos p(H-z) \cos k x,
$$

and from $(7),(19),(20)$ and (21),

$$
\rho^{\prime}=\left(s \cdot \sin p(H-z)-\frac{t k_{\lambda} p}{k_{\beta}{ }^{2}-k^{2}} \cos p(H-z)\right) h_{0} \cdot \frac{\cos k x}{\sin p H},
$$

where $t=\frac{1}{\rho_{0}} \frac{\partial \rho_{0}}{\partial y}$. In equations (19) to $(22), p$ should be understood as :

$$
p= \begin{cases}i \sqrt{ } k^{2}-k_{s}^{2}, & \text { for } k>k s, \\ \sqrt{\frac{k_{s}^{2}-k^{2}}{k^{2}-k_{\lambda}^{2}} \cdot k,} & \text { for } k_{s}>k>k_{\lambda}, \\ i k_{s} \sqrt{\frac{k^{2}-k_{\beta}{ }^{2}}{k_{\lambda}^{2}-k^{2}},} & \text { for } k_{\lambda}>k>k_{\beta}, \\ \frac{k_{s}}{k_{\lambda}} \sqrt{k_{\beta}{ }^{2}-k^{2}}, & \text { for } k<k_{\beta} .\end{cases}
$$

Thus we can easily verify that the wave length in the horizontal direction of the disturbances are the same as that of the bottom configuration, and when $k>k_{s}$, that is, when the bottom deformation is of very small scale, the amplitude of $v_{1}$ is very small compared with those of $u_{1}$ and $w_{1}$, and the disturbances may be considered to be confined to the $x-z$ plane. When $k_{s}>k>k_{\lambda}$, the amplitude of $v_{1}$ is still small compared with that of $u_{1}$, and the variation of velocities in the vertical direction is periodic if the condition, $k_{s}>\underset{H_{0}}{\pi}$, is satisfied. When $k_{\lambda}>k$ $>k_{\beta}$, that is, when bottom deformation is large, the amplitude of $v_{1}$ is large compared with that of $w_{1}$ and the disturbances may be considered to be almost

* Strictly speaking, small deformation of the free surface is expected, as in the case of usual internal waves. This effect, however, will be very small and may be neglected in this discussion. 
K. Kajiura: On the Influence of Bottom Topography on Ocean Currents.

horizontal, and when $k<k_{\beta}$, which is the case of a very large scale deformation, $w_{1} \ll v_{1}$ and the disturbances are almost horizontal so far as the depth of water, $H$, is not so great.

These solutions coincide with those obtained by Queney (1947) if the depth $H$ tends to infinity, and $k_{\beta}$ tends to zero.

In general, when the condition $|p H| \ll 1$ is fulfilled, (that is, $k<10^{-7} \mathrm{~cm}^{-1}$ for $H \sim 10^{5} \mathrm{~cm}, k_{s} \sim 10^{-4} \mathrm{~cm}^{-1}, k_{\lambda} \sim 10^{-5} \mathrm{~cm}^{-1}$ and $\left.k_{\beta} \sim 10^{-7} \mathrm{~cm}^{-1}\right)$ the disturbances may be simply written as:

$$
\begin{aligned}
& u_{1}=\frac{U h_{0}}{H} \cos k x, \\
& v_{1}=\frac{k k_{\lambda}}{k_{\beta}^{2}-k^{2}} \cdot \frac{U h_{0}}{H} \cdot k \sin k x, \\
& w_{1}=-\frac{H-z}{H} \cdot U k h_{0} \sin k x,
\end{aligned}
$$

and

$$
\rho^{\prime}=\frac{H-z}{H} \cdot s h_{0} \cos k x
$$

These solutions indicate that $u_{1}$ and $v_{1}$ may be considered as uniform in the vertical direction if the deformation of large scale is concerned. The above solutions can also be derived from (10) assuming that the water is homogeneous, $u_{1}$ and $v_{1}$ are independent of $z$, and $w_{1}$ is a linear function of $z$, satisfying the boundary conditions (17) and (18). In (30), the condition $\frac{s}{t} \gg \frac{k_{\lambda}(H-z)}{k_{\beta}{ }^{2}-k^{2}} \sim 10^{-4}$ is assumed, and the density distribution is just the same as suggested by Sverdrup. The effect of horizontal variation of density, however, is ineffective to the general pattern of the current, because we have assumed that the basic current is almost independent of the baroclinity of water. In this respect, we cannot discuss the influence of the density modification on the current pattern, which was the main part of Sverdrup's theory. From (20), it is found that the stream lines deviate to the left near the summit of the bottom deformation in the northern hemisphere if $k>k_{\beta}$ and just the opposite if $k<k_{\beta}$. This is illustrated in Fig. 1 .

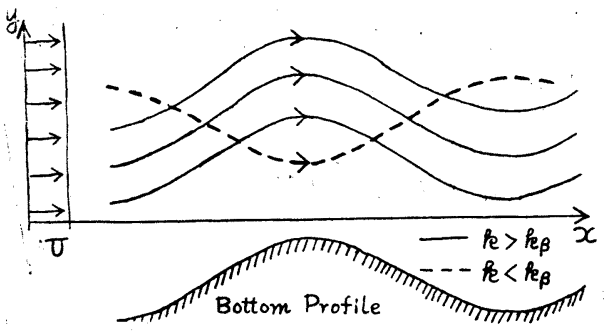

Fig. 1. Deflection of stream line by a sinusoidal bottom deformation in the case of $U=$ const. 


\section{Perturbations caused by the parabolic deformation of the bottom}

Let us now investigate the case when bottom deformation is such that

$$
h=0, \quad \text { for } \quad x \mid>a,
$$

and

$$
h=h_{0}\left(a^{2}-x^{2}\right), \text { for }|x|<a .
$$

We may write $h$ by the Fourier integral as follows:

$$
h=\frac{2 h_{0}}{\pi} \int_{-\infty}^{\infty} \frac{1}{k^{3}}(\sin k a-k a \cos k a) \cos k x \cdot d k .
$$

Then, from (16), (17) and (18), we have:

$$
w_{1}=-\frac{2 h_{0} U}{\pi} \int_{-\infty}^{\infty} \frac{\sin p(H-z)}{\sin p H} \cdot \frac{(\sin k a-k a \cos k a)}{k^{2}} \cdot \sin k x \cdot d k .
$$

Again, from (10) and (12), we have:

$$
v_{1}=\frac{2 h_{0} U}{\pi} \int_{-\infty}^{\infty} \frac{k_{\lambda}}{k_{\beta}{ }^{2}-k^{2}} \cdot \frac{p \cos p\left(H^{-}-z\right)}{\sin p H} \cdot \frac{(\sin k a-k a \cos k a)}{k^{2}} \cdot \sin k x \cdot d k .
$$

Both of these integrals can be evaluated by the method of contour integration in the complex plane.

Here we make the following transformations:

$$
\frac{\sin p(H-z)}{\sin p H}=\frac{2 \pi}{H^{2}} \sum_{n=1}^{n=\infty} \frac{n \sin \frac{\pi}{H} n z}{\left(n \frac{\pi}{H}\right)^{2}-p^{2}},
$$

and

$$
\frac{p \cos p(H-z)}{\sin p H}=-\frac{2 \pi^{2}}{H^{3}} \sum_{n=1}^{n-\infty} \frac{n^{2} \cos \frac{\pi}{H} n z}{\left(n \frac{\pi}{H}\right)^{2}-p^{2}}
$$

where $n$ is a positive integer. We may write approximately under the conditions $k_{s}^{2} \gg k_{\lambda}^{2} \gg k_{\beta}^{2}$ :

$$
\left(n \frac{\pi}{H}\right)^{2}=p^{2}=\left\{k^{2}\left(\begin{array}{c}
-\alpha_{n}^{2} \\
+\beta_{n}^{2}
\end{array}\right)\right\}\left\{k^{2}\left(\begin{array}{c}
+\gamma_{n}^{2} \\
-\delta_{n}^{2}
\end{array}\right)\right\} / k^{2}-k_{\lambda}^{2},
$$

where

and

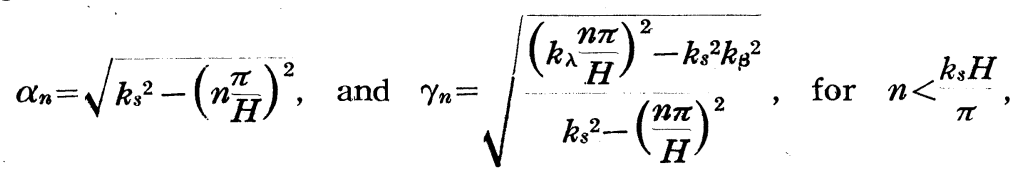

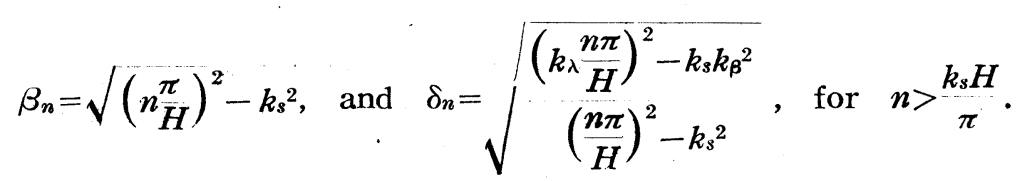


K. Kajiura: On the Influence of Bottom Topography on Ocean Currents.

In general, the conditions $\alpha_{n} \gg \gamma_{n}$ and $\beta_{n} \gg \delta_{n}$ are satisfied, and all these quantities are real.

Furthermore, we rewrite the terms of sine and cosine in exponential forms such as

$$
\begin{aligned}
& (\sin k a-k a \cos k a) \sin k x \\
& =-\frac{1}{4}\left[\left\{e^{i k(a+x)}-e^{i k(a-x)}-a k i\left(e^{i k(a+x)}-e^{i k(a-x)}\right)\right\}\right. \\
& \left.\quad+\left\{e^{-i k(a+x)}-e^{-i k(a-x)}+a k i\left(e^{-i k(a+x)}-e^{-i k(a-x)}\right)\right\}\right], \text { for }|x|<a,
\end{aligned}
$$

and

$$
\begin{aligned}
= & -\frac{1}{4}\left[\left\{e^{i k(x+a)}-e^{i k(x-a)}-a k i\left(e^{i k(x+a)}+e^{i k(x-a)}\right)\right\}\right. \\
& \left.+\left\{e^{-i k(x+x)}-e^{-i k(x-a)}+a k i\left(e^{-i k(x+a)}+e^{-i k(x-a)}\right)\right\}\right], \text { for }|x|>a .
\end{aligned}
$$

Thus, we must evaluate these integrals separately in each region, taking a suitable path of integration. The path may be taken from $-R$ to $R$, excluding poles, on the real axis and upper or lower semi-circle of radius $R$, so as to make the integral on the semi-circle to vanish when $R \rightarrow \infty$. If we take the principal values in Cauchy's sense, and substitute the conditions that $v^{\prime}=0$ and $w^{\prime}=0$ at $x=-\infty$, definite integral values can be obtained. (See Lamb, H. : Hydrodynamics. 6th Ed. P. 408)

The results are:

For $x<-a$,

$$
\begin{aligned}
v_{1}= & -2 h_{0} U\left\{\sum_{n=1}^{n=n_{0}} P_{\gamma n} e^{\gamma_{n} x}\left(\sinh \gamma_{n} a-a \gamma_{n} \cosh \gamma_{n} a\right)\right. \\
& \left.+\sum_{n=n_{0}+1}^{n=\infty} P_{\beta_{n}} e^{\beta_{n} x}\left(\sinh \beta_{n} a-a \beta_{n} \cosh \beta_{n} a\right)\right\},
\end{aligned}
$$

and

$$
\begin{aligned}
w_{1}= & -2 h_{0} U\left\{\sum_{n=\mathrm{L}}^{n=n_{0}} C_{n} e^{\gamma_{n} x}\left(\sinh \gamma_{n} \dot{a}-a \gamma_{n} \cosh \gamma_{n} a\right)\right. \\
& \left.+\sum_{n=n_{0}+1}^{n=\infty} B_{n} e^{\beta_{n} x}\left(\sinh \beta_{n} a-a \beta_{n} \cosh \beta_{n} a\right)\right\}
\end{aligned}
$$

For $-a<x<a$,

$$
\begin{aligned}
v_{1}=2 h_{0} U\left[P_{0} x-P_{1}\left\{\sin k_{\beta}(a+x)-a k_{\beta} \cos k_{\beta}(a+x)\right\}\right. \\
+\sum_{n=1}^{n=n_{0}}\left\{P_{\gamma n}\left(1+a \gamma_{n}\right) e^{-\gamma_{n} a} \sinh \gamma_{n} x\right. \\
\left.\quad-P_{\alpha n}\left(\sinh \alpha_{n}(a+x)-a \alpha_{n} \cos \alpha_{n}(a+x)\right)\right\} \\
+\sum_{n=n_{0}+1}^{n=\infty}\left\{P_{\beta n}\left(1+a \beta_{n}\right) e^{-\beta_{n} a} \sinh \beta_{n} x\right. \\
\left.-P_{\delta n}\left(\sinh \delta_{n}(a+x)-a \delta_{n} \cos \delta_{n}(a+x)\right\}\right]
\end{aligned}
$$


and

$$
\begin{aligned}
w_{1}=2 h_{v} U\left[P_{2} x+\sum_{n=1}^{n=n_{0}}\left\{C_{n}\left(1+a \gamma_{n}\right) e^{-\gamma_{n} a} \sinh \gamma_{n} x\right.\right. \\
\left.-A_{n}\left(\sin \alpha_{n}(a+x) a \alpha_{n} \cos \alpha_{n}(a+x)\right)\right\} \\
+\sum_{n=n_{0}+1}^{n=\infty}\left\{B_{n}\left(1+a \beta_{n}\right) e^{-\beta_{n} a} \sinh \beta_{n} x\right. \\
\left.\left.-D_{n}\left(\sin \delta_{n}(a+x)-a \delta_{n} \cos \delta_{n}(a+x)\right)\right\}\right] .
\end{aligned}
$$

For $x>a$,

$$
\begin{aligned}
v_{1}=2 h_{0} U\left[-2 P_{1} \cdot \cos k_{\beta} x\left(\sin k_{\beta} a-a k_{\beta} \cos k_{\beta} a\right)\right. \\
+\sum_{n=1}^{n=n_{0}}\left\{P_{\gamma n} e^{-\gamma_{n} x}\left(\sinh \gamma_{n} a-a \gamma_{n} \cosh \gamma_{n} a\right)\right. \\
\left.\quad-2 P_{\alpha n} \cos \alpha_{n} x\left(\sin \alpha_{n} a-a \alpha_{n} \cos \alpha_{n} a\right)\right\} \\
+\sum_{n=n_{0}+1}^{n=\infty}\left\{P_{\beta n} e^{-\beta_{n} x}\left(\sinh \beta_{n} a-a \beta_{n} \cosh \beta_{n} a\right)\right. \\
\left.\left.-2 P_{\delta n} \cos \delta_{n} x\left(\sin \delta_{n} a-a \delta_{n} \cos \delta_{n} a\right)\right\}\right],
\end{aligned}
$$

and

$$
\begin{array}{r}
w_{1}=2 h_{0} U\left[\sum _ { n = 1 } ^ { n = n _ { 0 } } \left\{C_{n} e^{-\gamma_{u} x}\left(\sinh \gamma_{n} a-a \gamma_{n} \cosh \gamma_{n} a\right)\right.\right. \\
\left.-2 A_{n} \cos \alpha_{n} x\left(\sin \alpha_{n} a-a \alpha_{n} \cos \alpha_{n} a\right)\right\} \\
+\sum_{n=n_{0}+1}^{n=\infty}\left\{B_{n} e^{-\beta_{n} x}\left(\sinh \beta_{n} a-a \beta_{n} \cosh \beta_{n} a\right)\right. \\
\left.\left.-2 D_{n} \cos \delta_{n} x\left(\sin \delta_{n} a-a \delta_{n} \cos \delta_{n} a\right)\right\}\right],
\end{array}
$$

where $n_{0}$ is the largest positive integer less than $k_{s} H / \pi$, and the following abbreviations are used:

$$
\begin{aligned}
& L_{n}=\frac{2 \pi n}{H^{2}} \sin \frac{\pi}{H} n z \\
& M_{n}=k_{\lambda} \cdot \frac{2 \pi^{2}}{H^{3}} \cos \frac{\pi}{H^{2} z} \\
& A_{n}=L_{n} \cdot \frac{1}{\alpha_{n}^{3}} \cdot \frac{\alpha_{n^{2}}{ }^{2}-k_{\lambda}^{2}}{\alpha_{n}^{2}-\gamma_{n}^{2}} \sim \frac{L_{n}}{\alpha_{n}^{3}}, \\
& B_{n}=L_{n} \cdot \frac{1}{\beta_{n}^{3}} \cdot \frac{\beta_{n}^{2}+k_{\lambda}^{2}}{\beta_{n}^{2}+\delta_{n}^{2}} \sim \frac{L_{n}}{\beta_{n}^{3}}, \\
& C_{n}=L_{n} \cdot \frac{1}{\gamma_{n}^{3}} \cdot \frac{\gamma_{n}^{2}+k_{\lambda}^{2}}{\gamma_{n}^{2}+\alpha_{n}^{2}}, \\
& D_{n}=L_{n} \cdot \frac{1}{\delta_{n}^{3}} \cdot \frac{\delta_{n}^{2}-k_{\lambda}^{2}}{\delta_{n}^{2}+\beta_{n}^{2}}, \\
& P_{0}=\frac{k_{s}}{k_{\beta}} \frac{\cos \left(k_{s} k_{\beta}(H-z) / k_{\lambda}\right)}{\sin \left(k_{s} k_{\beta} H / k_{\lambda}\right)} \sim \frac{k_{\lambda}}{k_{\beta}^{2} H},
\end{aligned}
$$


K. Kajiura: On the Influence of Bottom Topography on Ocean Currents.

$$
\begin{aligned}
& P_{1}=k_{\lambda} / k_{\beta}{ }^{3} H, \\
& P_{2}=-\frac{\sin \left(k_{s} k_{\beta}(H-z) / k_{\lambda}\right)}{\sin \left(k_{s} k_{\beta} H / k_{\lambda}\right)} \quad \sim-\frac{H-z}{H}, \\
& P_{\alpha n}=\frac{M_{n}}{L_{n}} \cdot \underset{\left(k_{\beta}{ }^{2}-\alpha_{n}^{2}\right)}{A_{n}} \quad \sim-\frac{M_{n}}{\alpha_{n}{ }^{5}}, \\
& P_{\beta n}=\frac{M_{n}}{L_{n}} \cdot \frac{B_{n}}{\left(k_{\beta}^{2}+\beta_{n}^{2}\right)} \sim \frac{M_{n}}{\beta_{n}{ }^{5}} \text {, } \\
& P_{\gamma n}=\frac{M_{n}}{L_{n}} \cdot \frac{C_{n}}{\left(k_{\beta}^{2}+\gamma_{n}^{2}\right)}, \\
& P_{\delta n}=\frac{M_{n}}{L_{n}} \cdot \frac{D_{n}}{\left(k_{\beta}^{2}-\delta_{n}^{2}\right)} .
\end{aligned}
$$

From these solutions, we can conclude that $\alpha_{n}$ and $\delta_{n}$, which exist only when $k_{s} H>\pi$, contribute to establish the stationary internal waves in the down-stream side of the deformation of the bottom, and no conspicuous disturbances occur in the up-stream side. And these internal waves prevail in the current pattern when the scale of deformation, $a$, is sufficiently small $\left(a k_{\lambda} \leqslant 1\right)$, on the other hand, they may be negligible compared with the horizontal perturbations when the scale of deformation is large. These features can be expected already from the discussion of perturbations for the sinusoidal bottom profile.

For $a k_{s} \ll 1$, the terms containing $\gamma_{n}$ and $\delta_{n}$ are negligibly small compared with those of $\alpha_{n}$ and $\beta_{n}$, and, at a sufficiently large distance downstream, the terms of $\alpha_{n}$ for $n$ is near $n_{0}$ predominate, since the terms of $\beta_{n}$ will die out. The leading term when $n_{0}=1$ is

$$
w_{1}=-\frac{4}{3} h_{0} U a^{3}\left(\frac{2 \pi}{H^{2}}\right) \sin \frac{\pi}{H} z \cos \alpha_{1} x,
$$

and the stream lines are given by

$$
\boldsymbol{P}=-\frac{4}{3} \frac{h_{0} a^{3}}{\alpha_{1}}\left(\frac{2 H}{H^{2}}\right) \sin \frac{\pi}{H} z \sin \alpha_{1} x .
$$

For $a k_{\lambda} \sim 1$, the terms of $\alpha_{n}$ and $\beta_{n}$ are rather small compared with the terms of $\gamma_{n}$ and $\delta_{n}$, so that in this case, the whole terms in the equations must be taken into account.

For $a k_{\lambda} \gg 1$, we may reasonably neglect the terms containing $\alpha_{n}, \beta_{n}, \gamma_{n}$ and $\delta_{n}$ and the solutions reduce to:

$$
\begin{aligned}
& v^{\prime}=0, \text { for } x<-a, \\
& v^{\prime}=-\frac{2 h_{0} U}{H} \cdot \frac{k_{\lambda}}{k_{\beta}{ }^{3}}\left\{\sin k_{\beta}(a+x)-a k_{\beta} \cos k_{\beta}(a+x)-k_{\beta} x\right\}, \text { for }-a<x<a
\end{aligned}
$$

and 


$$
v^{\prime}=-\frac{2 h_{0} U}{H} \cdot \frac{2 k_{\lambda}}{k_{\beta}{ }^{3}} \cos k_{\beta} x\left(\sin k_{\beta} a-a k_{\beta} \cos k_{\beta} a\right) \text {, for } x>a .
$$

These solutions can be derived from the original equations if the several assumptions are introduced such that the water is homogeneous and the variation of $u_{1}$ and $v_{1}$ in the vertical direction is negligible. In these solutions, we can see at once that in the northern hemisphere, the stream lines deviate to the right by the planetary effect and it is followed by Rossby waves. The deviation of stream line is illustrated qualitatively in Fig. 2.

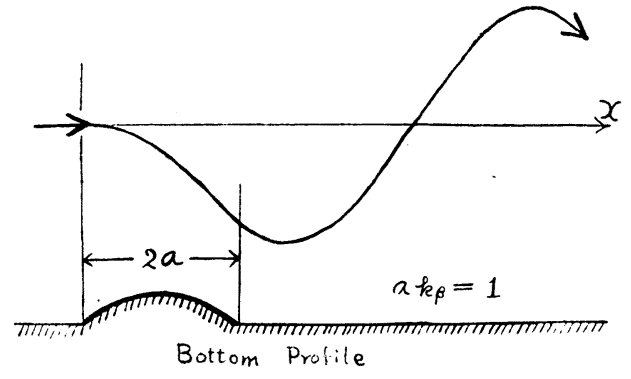

Fig. 2. Deflection of stream line by a large parabolic deformation of the bottom, ( $U=$ const.) $a=200 \mathrm{Km}$. $U=$ $40 \mathrm{~cm}^{-\mathrm{sec}^{-1},} k_{\beta}=5 \times 10^{-9} \mathrm{~cm}^{-1}$.

\section{The Effect of horizontal shear of a basic current.}

When the scale to be considered is large, and the basic current is constant in a vertical, we can assume from the above discussion that the horizontal velocities of perturbations are uniform in the vertical direction and the vertical velocity is a linear function of $z$. Therefore, on the assumption that the water is homogeneous and $\frac{\partial w_{1}}{\partial z}=-\frac{U}{H} \frac{d h}{d x}$, equation (8) becomes :

$$
\frac{\partial^{2} v_{1}}{\partial x^{2}}+\frac{\partial^{2} v_{1}}{\partial y^{2}}+\frac{1}{U}\left(\beta-\frac{d^{2} U}{d y^{2}}\right) v_{1}=-\frac{1}{H} \frac{d h}{d x}\left(\lambda-2 \frac{d U}{d y}\right)
$$

provided that $h$ is independent of $y$.

In a region of strong currents, it may be justified that $\beta$ is negligible compared with $d^{2} U / d y^{2}$. On these assumptions, we will discuss a very simple case, such as $U=U_{0} \cos k_{1} y$ and $h=h_{0} \cos k_{2} x$. A particular solution of the equation is then :

$$
v_{1}=\frac{h_{0}}{H}\left\{\begin{array}{c}
\lambda k_{2} \\
k_{1}{ }^{2}-k_{2}{ }^{2}
\end{array}-\frac{2 k_{1} U_{0}}{k_{2}} \sin k_{1} y\right\} \sin k_{2} x,
$$

where $H$ is the depth of water.

In case when $k_{1}$ is several times greater than $k_{2}, \quad\left(k_{1}=m k_{2}\right), k_{2}^{2}$ may be neglected compared with $k_{1}^{2}$, and (51) reduces to:

$$
v_{1}=\frac{h_{0}}{m H k_{1}}\left(\lambda-2 m^{2} U_{0} k_{1} \sin k_{1} y\right) \sin k_{2} x \text {. }
$$

From the above solution, we can see that it is very important to take the horizontal 
K. Kajiura: On the Influence of Bottom Topography on Ocean Currents.

shear of a current into account, if it is comparable with Coriolis parameter. The larger the ratio of the scale of the bottom deformation to that of currents is, the greater the effect of the shear is.

For example, in case of $U_{0} \sim$ $100 \mathrm{~cm} / \mathrm{sec}, k_{1} \sim 10^{-7} \mathrm{~cm}^{-1}\left(L_{1} \sim 600\right.$ $\mathrm{km})$, and $k_{2} \sim 3.3 \times 10^{-8}\left(L_{2} \sim 2000\right.$ $\mathrm{km}), m$ becomes 3 and the order of magnitude of $m^{2} U_{0} k_{1}$ is almost comparable with that of $\lambda\left(\sim 10^{-4} \mathrm{sec}^{-1}\right)$. The current pattern is shown qualitatively in Fig. 3.

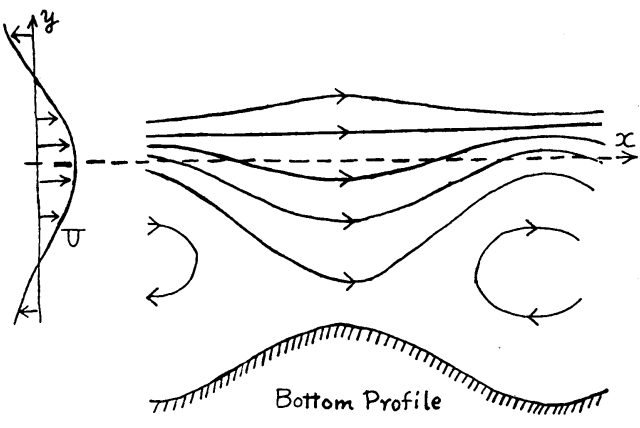

Fig. 3. Deflection of stream lines by a sinusoidal bottom deformation in the case of $U=U_{0} \cos k_{1} y, h=h_{0} \cos k_{2} y$, and $\lambda=\mathrm{m}^{2} U_{0} k_{1}$.

\section{Conclusions.}

We have discussed above that when we are investigating the effect of bottom topography on a uniform basic current, it is important to consider the relation of the scale of the bottom configuration to various parameters such as $k_{s}, k_{\lambda}$ and $k_{\beta}$, each of which is the representative scale of the motion of the stratified water on the rotating earth. When the scale of bottom deformations are very small, such that $k_{\lambda} a \ll 1$, the parameter $k_{s}$, which is related to the vertical gradient of the density, plays a dominant role and the perturbation may be almost two dimensional in the vertical plane, and, moreover, if $k_{s} H>\pi$, the internal gravity waves occur downstream, the wave length of which is $2 H^{\mathrm{o}} / n$ in the vertical direction and $2 \pi / \alpha_{n}$ in the horizontal direction.

When the scale of bottom deformations is very large such that $k_{\lambda} a \gg 1$, the parameter $k_{\beta}$, which is related to the latitudinal change of Coriolis parameter, plays a dominant role and the conclusions are the same as those obtained on the assumption of homogeneous water, such that the stream-lines deviate to the right in the northern hemisphere, accompanied by the Rossby waves.

In the case of an intermediate scale of phenomena, the parameter $k_{\lambda}$, which is related to Coriolis parameter itself, must be taken into account and the perturbations are considered as the combined effects of various factors.

On the other hand, it was shown that, if the basic current is not uniform, the horizontal shear of velocity may sometimes play an important role in the discussion of the topographic effect upon the currents. Thus, the deflection of 
currents may be related to the horizontal gradient of velocity and Coriolis parameter.

It must be remembered, however, that the whole discussions are made under the assumption that the steady perturbations always exist, regardless of values of various parameters, so that the discussions of stability and instability of these flow patterns are entirely ommitted.

\section{Acknowledgements.}

The author is indebted to Mr. H. Kusunoki, of the Low Temperature Science Institute, Hokkaido Univ., who generously communicated him the papers of V. W. Stockman. He also wishes to thank Dr. M. Nakano, who kindly read the manuscript before printing.

\section{References}

Bolin, B., 1950: On the influence of the earth's orography on the general character of the westerlies. Tellus, 2, (3), pp. 184-195.

Charney, J. and Eliassen, A., 1949: A numerical method for predicting the perturbations of the middle latitude westerlies. Tellus, 1, (2), pp. 38-54.

Colson, D., 1949: Air flow over a mountain barrier. Trans. Amer. Geophys. Union, 30, pp. $818-830$.

Ekman, V. W., 1923: Über Horizontalzirkulation bei winderzeugten Meeresströmungen. Ark. f. Mat., Astr. och Fys., Bd. 17, No. 26.

Ekman, V. W., 1932: Studien zur Dynamik der Meeresströmungen. Beitr. zur Geophys., Bd, 36, S. 385-438.

Queney, P., 1947: Theory of Perturbations in Stratified Currents with Applications to Air Flow over Mountain Barriers. Misc. Report No. 23, University of Chicago.

Queney, P., 1948: The problem of air flow over mountains; a summary of theoretical studies. Bull. Amer. Met. Soc., 29, (1), pp. 16-26.

Stockman, V. B., 1947: Effect of Bottom Topography on the Direction of Currents in the Sea. Priroda, 11, pp. 10-23. (Defence Research Board, Canada., T 57 R.)

Stockman, V. B., 1948: Effect of bottom topography on the direction of the transport of water set up by the wind or the muss-field in a non-homogeneous ocean. Dok. Acad. Nauk. SSSR, 59, (5), pp. 889-892. (DRB, Canada. T 56 R.)

Sverdrup, H. U., 1941: The influence of bottom topography on ocean currents. Applied Mechanics, Th. von Kármán Anniv. Volume. 